\title{
Determination of the levels of tryptophan and 12 metabolites in milk by liquid chromatography-tandem mass spectrometry with the QuEChERS method
}

\author{
Meicheng Su, ${ }^{1 *} \odot$ Yongyou Cheng, ${ }^{2 *}$ Congcong Zhang, ${ }^{1}$ Dan Zhu, ${ }^{1}$ Man Jia, ${ }^{1}$ Qingyang Zhang, ${ }^{1}$ Huaxing Wu, ${ }^{3,4}$ \\ and Gang Chen ${ }^{1}+$ (iD \\ ${ }^{1}$ Key Laboratory of Agro-product Quality and Safety, Institute of Quality Standard \& Testing Technology for Agro-Products, \\ Chinese Academy of Agricultural Sciences, Beijing, China 100081 \\ ${ }^{2}$ College of Food and Pharmaceutical Engineering, Guiyang University, Guizhou Province 550004, China \\ ${ }^{3}$ R\&D Center, New Hope Dairy Co, Ltd. Chengdu 610000, China \\ ${ }^{4}$ Dairy Nutrition and Function, Key Laboratory of Sichuan Province, Chengdu, 610000, China
}

\begin{abstract}
Tryptophan and metabolites have important biological functions in humans. Milk is an important source of tryptophan intake. In this study, we developed a method to detect levels of tryptophan and 12 metabolites in milk. The analytes were extracted by using the QuEChERS (quick, easy, cheap, effective, rugged, and safe) procedure and analyzed by liquid chromatography-tandem mass spectrometry with electrospray ionization. The proposed method resulted in suitable accuracy (standard deviation $\leq 10.31 \%$ ) and high sensitivity (the limits of quantification were between 0.05 and $5 \mathrm{ng} / \mathrm{mL}$ ). Recoveries were in the range of 44 to $126 \%$. Finally, the developed method was successfully applied to compare the content of tryptophan and metabolites in 4 milk products produced by different processes: pasteurized milk, UHT milk, milk powder, and yogurt. The results of partial least squares-discriminant analysis (PLS-DA) showed that different types of processed milk could be distinguished clearly according to the method used here. The determined tryptophan and metabolites levels in milk can provide a new reference for evaluation of milk.
\end{abstract}

Key words: tryptophan metabolite, milk, liquid chromatography-tandem mass spectrometry

\section{INTRODUCTION}

Tryptophan is 1 of 8 essential amino acids required by the human body. It not only participates in the synthesis of protein in the body but also affects mental stability and depression (Sonner et al., 2019). In addition, tryptophan can be metabolized into many bioac-

Received January 26, 2020.

Accepted June 26, 2020.

*These authors contributed equally to this work.

†Corresponding author: chengang01@caas.cn tive substances. Various aspects of the biological function of tryptophan metabolites have been illustrated, especially in the medical field, where tryptophan and metabolites are used to indicate the appropriate treatments of many diseases (Arvidsson et al., 2007; Kato et al., 2007; Henykova et al., 2016; Marcos et al., 2016; Guo et al., 2017). The metabolism of tryptophan follows 3 main pathways. The first is the kynurenine pathway, which forms kynurenine acid, niacin, and xanthurenic acid; kynurenine acid has a neuroprotective effect and can resist the neurotoxicity caused by excessive excitability of quinolinic acid (Chen and Guillemin, 2009). Niacin, the final product of this pathway, has antiinflammatory and antioxidant properties, which may be beneficial in treating obesity and atherosclerosis. The second is the serotonin pathway, which produces serotonin, melatonin, and 5-hydroxytryptophan; these metabolites can improve sleep, reduce irritability, and bring feelings of happiness. The third pathway is the indole pathway, which forms indole formaldehyde, indole acetic acid, indole-3-acetamide, and tryptamine, among others; some of these metabolites have immune regulation functions.

Tryptophan and metabolites exist in a wide variety of diets. Tryptophan and metabolites have been detected in vegetables (Turski et al., 2011), honey (Soto et al., 2011), spices (Turski et al., 2015), and wine (Mattivi et al., 1999). However, as far as we know, only a few studies have reported on tryptophan and its metabolites in milk (Bertazzo et al., 2016; Yilmaz and Gokmen, 2018). Studies on the different types of tryptophan metabolites are limited and have focused on only a few compounds without focusing on their biological functions. Considering that milk is one of the most important sources for tryptophan intake, it is necessary to investigate the contents of bioactive tryptophan metabolites in milk and provide references for milk nutritional evaluation.

In this work, we developed a new method to determine tryptophan and 12 metabolites in milk. We used a fully 
developed QuEChERS (quick, easy, cheap, effective, rugged, and safe) sample extraction procedure, which enabled more tryptophan metabolites to be analyzed, compared with previously published results (Kocadagli et al., 2014; Bertazzo et al., 2016). The QuEChERS method had high sensitivity and can be practically applied to real sample analysis. We used this method to measure levels of bioactive tryptophan metabolites in milk from different processing methods.

\section{MATERIALS AND METHODS}

\section{Chemicals and Reagents}

All reagents were of analytical grade unless otherwise stated. Melatonin (MT), 5-hydroxytryptophan (5-HTP), serotonin (5-HT), indole-3-acetamide (IAM), tryptamine ( $\operatorname{Tr} \mathbf{A})$, 3-hydroxyanthranilic acid (3-HAA), niacin, xanthurenic acid (XA), tryptophan (Trp), kynurenine (KYN), kynurenine acid (KA), indole-3-carboxaldehyde (IAId), and indole-3-acetic acid (IAA) were purchased from Sigma-Aldrich (Darmstadt, Germany); the purity of these standards was $>99 \%$. Bondesil $\mathrm{C}_{18}$ sorbent (average particle size $40 \mu \mathrm{m}$ ) was purchased from Agilent Technologies Inc. (Santa Clara, CA), and $\mathrm{Na}_{2} \mathrm{SO}_{4}$ and $\mathrm{NaCl}$ were purchased from Sinopharm Chemical Reagent Co. Ltd. (Shanghai, China). Water was purified by using a Milli-Q system from Millipore (Bedford, MA). Formic acid, methanol $(\mathbf{M e O H})$, and acetonitrile (ACN) were HPLC grade and purchased from Fisher Scientific (Pittsburgh, PA).

\section{Sample Collection and Preparation}

Five brands of pasteurized milk, 10 brands of UHT whole milk, 10 brands of milk powder, and 10 brands of yogurt were purchased from local supermarkets in China. Each brand included 5 samples with different production dates (see Supplemental Table S1; https: //doi.org/10.3168/jds.2020-18260). The samples were stored at $-20^{\circ} \mathrm{C}$ in the dark until the analysis was performed.

We adapted and optimized a previously published QuEChERS procedure (Wilkowska and Biziuk, 2011; Costa et al., 2014) that was used to analyze pesticide residues in food as follows: $5 \mathrm{~mL}$ of a liquid milk sample was added to a centrifuge tube, and $10 \mathrm{~mL}$ of ACN containing $5 \%$ formic acid was added. The mixture was stirred for $10 \mathrm{~min}$ at room temperature, and $1 \mathrm{~g}$ of $\mathrm{NaCl}$ and $4 \mathrm{~g}$ of $\mathrm{Na}_{2} \mathrm{SO}_{4}$ were added to promote salt-assisted liquid-liquid extraction, which was followed by stirring for $2 \mathrm{~min}$ and centrifugation at $10,000 \times g$ for $10 \mathrm{~min}$. Seven milliliters of the upper layer was transferred to another centrifuge tube, and a dispersive solid-phase extraction (150 mg of $\mathrm{C}_{18}+900 \mathrm{mg}$ of $\mathrm{Na}_{2} \mathrm{SO}_{4}$ ) was added. After stirring for $2 \mathrm{~min}$ and centrifugation at $10,000 \times g$ for $10 \mathrm{~min}, 6 \mathrm{~mL}$ of supernatant was taken and evaporated until dry under nitrogen protection and reconstituted with $1 \mathrm{~mL}$ of $\mathrm{ACN}: \mathrm{H}_{2} \mathrm{O}$ (10:90, vol/vol) for the liquid chromatography-tandem mass spectrometry (LC-MS/MS) analysis.

Milk powder was reconstituted with hot water at a ratio of 1:6 to achieve a protein content of $3.0 \mathrm{~g} / \mathrm{mL}$. The remaining product types followed the process described above. The yogurt samples were diluted to 1:1 ( $\mathrm{vol} / \mathrm{vol}$ ) and extracted following the same procedure as used for the liquid milk. All samples were analyzed in triplicate.

\section{Instrumentation and Software}

The LC-MS/MS was carried out on an Acquity UPLC (Waters, Milford, MA) system and Qtrap 4500 mass spectrometry (AB Sciex, Toronto, ON, Canada) with an ESI source. A Zorbax Eclipse plus $\mathrm{C}_{18}$ column $(150 \mathrm{~mm} \times 3.0 \mathrm{~mm}$ i.d., $1.8 \mu \mathrm{m}$ particle $)$ from Agilent Technologies Inc. was used for chromatographic separation at a flow rate of $0.3 \mathrm{~mL} / \mathrm{min}$. The column temperature was set at $30^{\circ} \mathrm{C}$ and the injection volume was $5 \mu \mathrm{L}$. Mobile phase A was water with $0.1 \%$ formic acid, and mobile phase $\mathrm{B}$ was $\mathrm{MeOH}$ with $0.1 \%$ formic acid. The gradient program of mobile phase $\mathrm{B}$ was set as follows: $0-1 \mathrm{~min}, 5 \% \mathrm{~B} ; 1-1.5 \mathrm{~min}, 5-15 \% \mathrm{~B} ; 1.5-2$ min, $15 \%$ B; 2-3.5 min, 15-70\% B; 3.5-4.5 min, $70 \%$ B; 4.5-5.5 min, 70-90\% B; 5.5-7.5 min, $90 \%$ B; 7.5-8 min, 90-5\% B; 8-10 min, 5\% B.

All compounds were determined by multiple reaction monitoring in positive ionization mode. The MS conditions were as follows: curtain gas, 35 psi; collision gas, $10 \mathrm{psi}$; ion spray voltage $5,500 \mathrm{~V}$; temperature, $500^{\circ} \mathrm{C}$; ion source gas $1,50 \mathrm{psi}$; ion source gas 2,50 psi. The declustering potential and collision energy of each compound were optimized. The transition information and optimized parameters for each compound are listed in Table 1.

\section{Method Validation}

The tryptophan and metabolites in the samples were calculated by matrix-matched calibration curves, which were prepared by spiking the extracted samples with standard solutions. The concentration of metabolites in the blank milk was subtracted for the recovery calculation. The limit of detection (LOD) and limit of quantification (LOQ) were determined when the signal-to-noise ratios were 3 and 10, respectively. The 
Table 1. Mass spectrometry parameters for tryptophan and metabolite analysis ${ }^{1}$

\begin{tabular}{|c|c|c|c|c|}
\hline Compound & $\begin{array}{l}\text { Precursor } \\
\text { ion }(m / z)\end{array}$ & $\begin{array}{l}\text { Product } \\
\text { ion }(m / z)\end{array}$ & $\begin{array}{l}\mathrm{DP} \\
(\mathrm{V})\end{array}$ & $\begin{array}{c}\mathrm{CE} \\
(\mathrm{eV})\end{array}$ \\
\hline Melatonin (MT) & 232.9 & $174.1^{2}$ & 71 & 17 \\
\hline \multirow[t]{2}{*}{ 5-Hydroxytryptophan (5-HTP) } & 220.9 & $\begin{array}{l}159.1 \\
204.1^{2}\end{array}$ & $\begin{array}{l}67 \\
60\end{array}$ & $\begin{array}{l}40 \\
16\end{array}$ \\
\hline & & 162.0 & 60 & 25 \\
\hline \multirow[t]{2}{*}{ Serotonin (5-HT) } & 176.8 & $160.0^{2}$ & 60 & 13 \\
\hline & & 115.0 & 60 & 36 \\
\hline \multirow[t]{2}{*}{ Indole-3-acetamide (IAM) } & 175.1 & $130.1^{2}$ & 75 & 15 \\
\hline & & 103.0 & 75 & 46 \\
\hline \multirow[t]{2}{*}{ Tryptamine (TrA) } & 160.9 & $144.1^{2}$ & 52 & 12 \\
\hline & & 117.0 & 52 & 35 \\
\hline \multirow[t]{3}{*}{ 3-Hydroxyanthranilic acid (3-HAA) } & 154.1 & 136.0 & 60 & 15 \\
\hline & & $108.1^{2}$ & 54 & 29 \\
\hline & & 80.1 & 58 & 32 \\
\hline \multirow[t]{2}{*}{ Niacin } & 124.0 & $78.0^{2}$ & 60 & 33 \\
\hline & & 53.0 & 60 & 39 \\
\hline \multirow[t]{2}{*}{ Xanthurenic acid (XA) } & 206.0 & $160.0^{2}$ & 75 & 24 \\
\hline & & 132.1 & 75 & 40 \\
\hline \multirow[t]{2}{*}{ Tryptophan (Trp) } & 205.1 & $188.1^{2}$ & 60 & 13 \\
\hline & & 146.0 & 65 & 20 \\
\hline \multirow[t]{3}{*}{ Kynurenine (KYN) } & 208.9 & 192.1 & 60 & 13 \\
\hline & & $94.1^{2}$ & 60 & 19 \\
\hline & & 146.1 & 56 & 29 \\
\hline \multirow[t]{2}{*}{ Kynurenine acid (KA) } & 189.9 & $144.1^{2}$ & 95 & 24 \\
\hline & & 116.0 & 90 & 44 \\
\hline \multirow[t]{2}{*}{ Indole-3-carboxaldehyde (IAld) } & 146.2 & $118.1^{2}$ & 55 & 20 \\
\hline & & 91.1 & 55 & 30 \\
\hline \multirow[t]{2}{*}{ Indole-3-acetic acid (IAA) } & 176.1 & $130.1^{2}$ & 64 & 27 \\
\hline & & 103.0 & 68 & 43 \\
\hline
\end{tabular}

${ }^{1} \mathrm{DP}=$ declustering potential; $\mathrm{CE}=$ collision energy.

${ }^{2}$ Quantification ion.

repeatability and precision of the methods were determined by spiking samples with standard solutions at 3 concentrations ( 5 replicates for each level) and testing for 3 consecutive days. Standard deviations (SD\%) were used to evaluate precision.

\section{Statistical Analysis}

The raw data were imported into the Multiquant software (version 5.0, AB Sciex) to calculate the concentrations of the compounds. The generated data were exported as an XML file using Excel 2016 (Microsoft Corp., Redmond, WA) and then processed using SPSS Statistics 25.0 (IBM Corp., Armonk, NY) for the ANOVA. The SIMCA 14.1.0 software (Umetrics, Umea, Sweden) was used for the partial least squaresdiscriminant analysis (PLS-DA).

\section{RESULTS AND DISCUSSION}

\section{Method Optimization}

$L C-M S / M S$ Conditions. As shown in Figure 1, all of the compounds were ionized as $\left[\mathrm{M}+\mathrm{H}^{+}\right]$, because the $\mathrm{N}$ atom existed in their molecular structure. The precursor and product ions were monitored in positive ionization mode. To obtain the highest sensitivity, the precursor ions were optimized at declustering potential values of 20 to $120 \mathrm{~V}$, and the product ions were optimized at collision energy values of 10 to $80 \mathrm{~V}$. The ion information, as well as the optimized declustering potential and collision energy parameters, are shown in Table 1.

$\mathrm{A} \mathrm{C}_{18}$ column was selected for chromatographic separation. Compared with $\mathrm{ACN}$ (0.1\% formic acid), $\mathrm{MeOH}$ ( $0.1 \%$ formic acid) gave higher intensity and a sharper peak and greater separation for analytes; therefore, it was selected as the organic mobile phase. A gradient program was optimized based on a shallow gradient to obtain satisfactory separation among the peaks.

Sample Preparation. The sample preparation procedure was optimized by spiking dairy samples with the standard solution. Both solid-phase extraction cartridges [Oasis HLB (Thermo Fisher Scientific, Waltham, MA), Sep-Pak C18 (Agilent Technologies Inc.), Oasis MCX (Thermo Fisher Scientific), and Bond Elut Plexa PCX (Agilent Technologies Inc.)] and the QuEChERS method were tested for cleanup. We found 


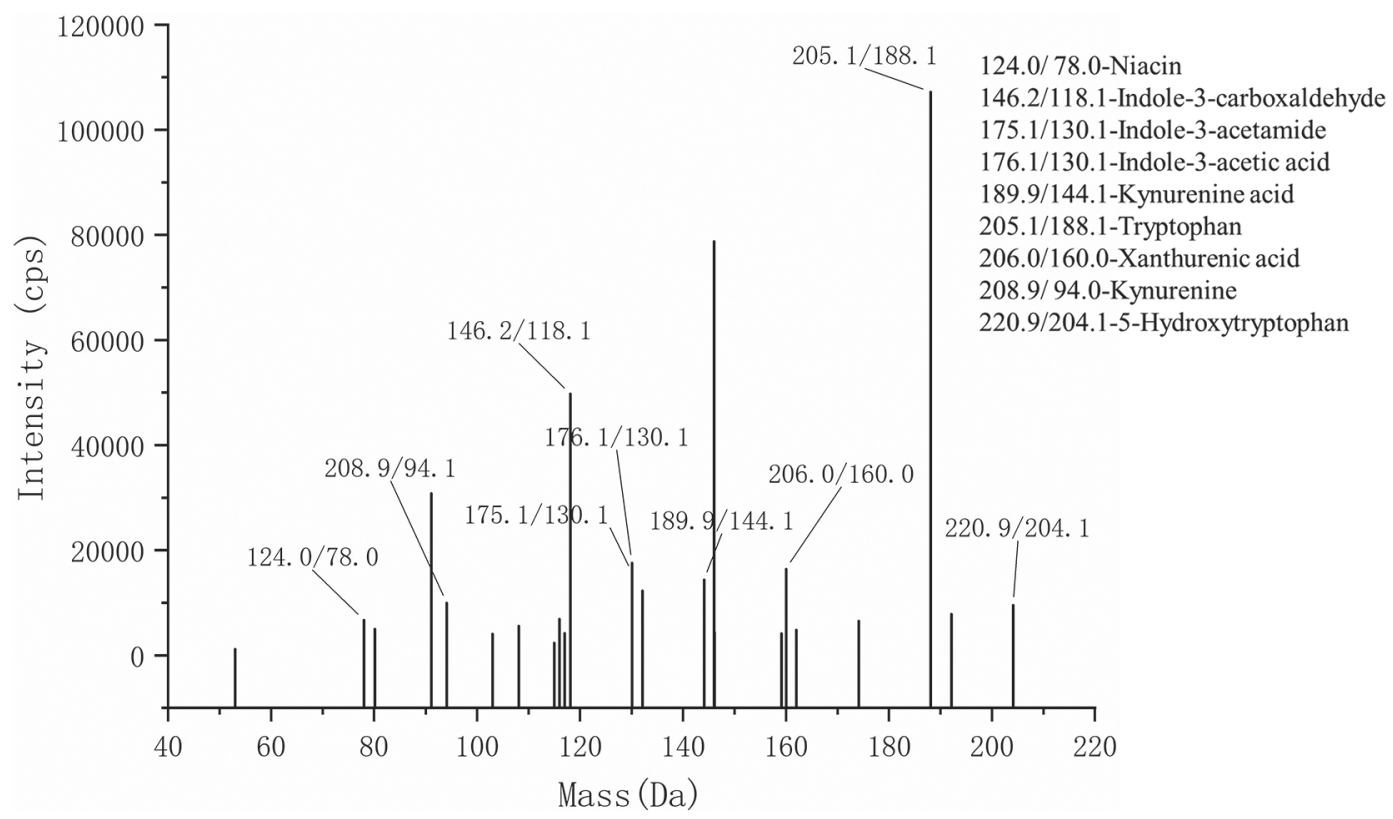

Figure 1. Mass spectrogram of the tryptophan and metabolites in the milk samples.

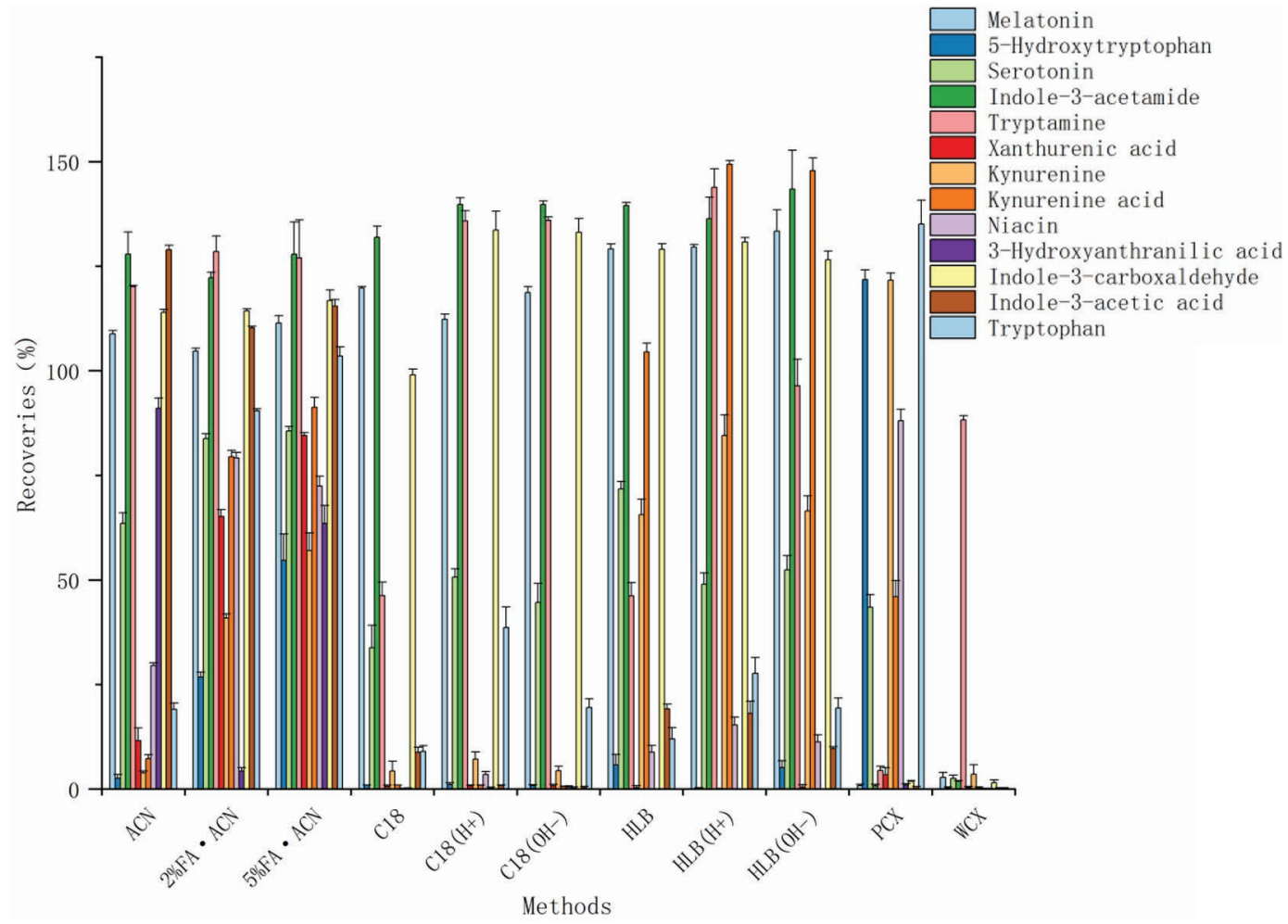

Figure 2. Recoveries of tryptophan and metabolites in milk by different solid-phase extraction cartridges and QuEChERS methods. The tryptophan and metabolites were spiked at $50 \mathrm{ng} / \mathrm{mL}$ in the milk matrix. $\mathrm{H}^{+}$: eluent is methanol containing $5 \%$ formic acid (FA); $\mathrm{OH}^{-}$: eluent is methanol containing 5\% ammonia; ACN = acetonitrile; C18 = Sep-Pak C18 (Agilent Technologies Inc., Santa Clara, CA); HLB = Oasis HLB (Thermo Fisher Scientific, Waltham, MA); PCX = Bond Elut Plexa PCX (Agilent Technologies Inc.); WCX = Oasis MCX (Thermo Fisher Scientific). 
Table 2. The limit of detection (LOD) and limit of quantification (LOQ) values of the tryptophan and metabolites in the milk samples from the 4 processing technologies $(\mathrm{ng} / \mathrm{mL})$

\begin{tabular}{|c|c|c|c|c|c|c|c|c|}
\hline \multirow[b]{2}{*}{ Compound $^{1}$} & \multicolumn{2}{|c|}{ Pasteurized milk } & \multicolumn{2}{|c|}{ UHT milk } & \multicolumn{2}{|c|}{ Reconstituted milk } & \multicolumn{2}{|c|}{ Yogurt } \\
\hline & LOD & LOQ & LOD & LOQ & LOD & LOQ & LOD & LOQ \\
\hline MT & 0.02 & 0.04 & 0.02 & 0.05 & 0.02 & 0.06 & 0.02 & 0.05 \\
\hline 5-HTP & 0.01 & 0.02 & 0.01 & 0.03 & 0.02 & 0.06 & 0.02 & 0.04 \\
\hline 5-HT & 0.01 & 0.02 & 0.05 & 0.20 & 0.01 & 0.04 & 0.02 & 0.06 \\
\hline IAM & 0.02 & 0.04 & 0.02 & 0.07 & 0.04 & 0.15 & 0.03 & 0.10 \\
\hline TrA & 0.01 & 0.04 & 0.02 & 0.04 & 0.02 & 0.07 & 0.02 & 0.05 \\
\hline XA & 0.01 & 0.02 & 0.02 & 0.04 & 0.03 & 0.10 & 0.02 & 0.08 \\
\hline Trp & 0.01 & 0.04 & 0.01 & 0.03 & 0.02 & 0.04 & 0.01 & 0.04 \\
\hline KYN & 0.01 & 0.03 & 0.01 & 0.02 & 0.01 & 0.02 & 0.01 & 0.03 \\
\hline $\mathrm{KA}$ & 0.02 & 0.05 & 0.01 & 0.03 & 0.01 & 0.02 & 0.03 & 0.90 \\
\hline Niacin & 0.08 & 0.24 & 0.01 & 0.03 & 0.10 & 0.30 & 0.04 & 0.15 \\
\hline 3 -HAA & 0.05 & 0.16 & 0.14 & 0.46 & 0.10 & 0.35 & 2.00 & 5.00 \\
\hline IAld & 0.02 & 0.04 & 0.01 & 0.03 & 0.02 & 0.05 & 0.02 & 0.05 \\
\hline IAA & 0.02 & 0.08 & 0.01 & 0.03 & 0.03 & 0.10 & 0.04 & 0.15 \\
\hline
\end{tabular}

${ }^{1} \mathrm{MT}=$ melatonin; 5 -HTP $=$ 5-hydroxytryptophan; 5-HT = serotonin; IAM $=$ indole-3-acetamide; $\mathrm{TrA}=$ tryptamine; 3 -HAA = 3-hydroxyanthranilic acid; XA = xanthurenic acid; Trp = tryptophan; KYN = kynurenine; $\mathrm{KA}=$ kynurenine acid; IAId = indole-3-carboxaldehyde; IAA = indole-3-acetic acid.

that the 13 analytes could not be completely retained or eluted in the 4 cartridges (Figure 2). In contrast, the QuEChERS method provided acceptable recoveries for all analytes. Moreover, to optimize the extraction solvent in QuEChERS, ACN solvents with different concentrations of formic acid ( 0,2 , and $5 \%$ formic acid) were tested, and $5 \%$ formic acid in ACN resulted in the highest recoveries for all analytes (Figure 2).

\section{Method Validation}

Calibration. The matrix interference was evaluated by comparing the results of the standards spiked in the sample matrix with a neat standard solution (Sadok et al., 2019). Matrix-assisted standard curves were used for quantification of the analytes because matrix interference cannot be totally removed by sample extraction. Seven concentrations of each compound were used to construct the calibration curve. The linear range, regression equation, and $\mathrm{R}^{2}$ values are shown in Supplemental Table S2 (https://doi.org/10.3168/jds.2020 -18260). The $\mathrm{R}^{2}$ of each calibration curve was $>0.99$.

Sensitivities. The LOD and LOQ of the analytes in 4 types of milk are shown in Table 2 . Very high sensitivities $(<1 \mathrm{ng} / \mathrm{mL})$ were achieved for most analytes, except that the LOD and LOQ of 3-HAA in yogurt were 2 and $5 \mathrm{ng} / \mathrm{mL}$, respectively. The performance of the present method was compared with previously

Table 3. Comparison of the method performance (recovery, limits of detection and quantification) in the present work and in the literature

\begin{tabular}{|c|c|c|c|c|c|c|c|c|c|}
\hline \multirow[b]{2}{*}{ Compound $^{1}$} & \multicolumn{3}{|c|}{ Recovery (\%) } & \multicolumn{3}{|c|}{ Limit of detection $(\mathrm{ng} / \mathrm{mL})$} & \multicolumn{3}{|c|}{ Limit of quantification $(\mathrm{ng} / \mathrm{mL})$} \\
\hline & $\begin{array}{l}\text { Present } \\
\text { work }\end{array}$ & $\begin{array}{l}\text { Yllmaz and } \\
\text { Gokmen } \\
(2018)\end{array}$ & $\begin{array}{l}\text { Soto et al. } \\
\quad(2011)\end{array}$ & $\begin{array}{c}\text { Present } \\
\text { work }\end{array}$ & $\begin{array}{l}\text { Yllmaz and } \\
\text { Gokmen } \\
(2018)\end{array}$ & $\begin{array}{l}\text { Soto et al. } \\
\quad(2011)\end{array}$ & $\begin{array}{c}\text { Present } \\
\text { work }\end{array}$ & $\begin{array}{l}\text { Yllmaz and } \\
\text { Gokmen } \\
(2018)\end{array}$ & $\begin{array}{c}\text { Soto et al. } \\
\quad(2011)\end{array}$ \\
\hline $\mathrm{MT}$ & $75.31-104.39$ & - & - & 0.02 & - & - & $0.05-0.06$ & - & - \\
\hline 5-HTP & $44.67-55.69$ & - & - & $0.01-0.02$ & - & - & $0.02-006$ & - & - \\
\hline 5 -HT & $74.87-89.19$ & - & - & $0.01-0.05$ & - & - & $0.02-0.2$ & - & - \\
\hline XA & $78.38-99.93$ & - & $41.2-134.2$ & $0.01-0.03$ & - & $0.6-1.0$ & $0.02-0.1$ & - & $1.9-3.4$ \\
\hline $\operatorname{Trp}$ & $95.60-101.61$ & $88.4-109.2$ & $38.3-116.7$ & $0.01-0.02$ & $1.4-37$ & $0.2-0.5$ & $0.03-0.04$ & $4.7-123.5$ & $0.6-1.7$ \\
\hline KYN & $56.00-80.94$ & $59.3-124.1$ & $31.5-144.3$ & $0.01-0.02$ & $0.5-2$ & $0.5-0.8$ & $0.02-0.03$ & $1.7-6.7$ & $1.9-3.5$ \\
\hline KA & $77.27-103.56$ & $72.9-114.5$ & $46.8-114.3$ & $0.01-0.03$ & $0.1-4.8$ & $0.5-0.9$ & $0.05-0.9$ & $0.3-15.9$ & $1.5-3.0$ \\
\hline Niacin & $55.10-88.50$ & $72.0-99.2$ & - & $0.01-0.1$ & $0.9-31.4$ & - & $0.03-0.3$ & $2.9-94.5$ & - \\
\hline 3 -HAA & $55.82-90.00$ & - & - & $0.05-2$ & - & - & $0.16-5$ & - & - \\
\hline IAld & $100.59-127.86$ & - & - & $0.01-0.02$ & - & - & $0.03-0.05$ & - & - \\
\hline
\end{tabular}

${ }^{1} \mathrm{MT}=$ melatonin; 5-HTP $=$ 5-hydroxytryptophan; 5-HT $=$ serotonin; IAM $=$ indole-3-acetamide; TrA = tryptamine; 3-HAA $=3$-hydroxyanthranilic acid; XA = xanthurenic acid; Trp = tryptophan; KYN = kynurenine; KA = kynurenine acid; IAId = indole-3-carboxaldehyde; IAA $=$ indole-3-acetic acid. 
reported methods. Most studies only focused on a few compounds, primarily KYN, KA, niacin, 3-HAA, XA, and 5-HT. Additionally, the sensitivities in our study were 20 to 100 times higher than the reported results (see the comparison in Table 3 ).

Repeatability and Precision. The repeatability and precision of the methods were assessed by spiking real samples with standard solutions at 3 concentrations in 5 replicates for each sample and testing for 3 consecutive days. As shown in Table 4, recoveries were between 50 and $126 \%$, except for 5 -HTP in reconstituted milk, which was 44 to $50 \%$. All of the standard deviations were $<10.31 \%$, demonstrating that the developed method has suitable precision.

\section{Method Application}

The contents of tryptophan and its metabolites in 4 types of milk (pasteurized, UHT, milk powder, and yogurt) were analyzed by the developed method. All 13 analytes could be detected, except that 3-HAA was not detectable in yogurt (Table 5). The tryptophan concentrations ranged from $8,960.70$ to $11,771.40 \mathrm{ng} / 100$ $\mathrm{mL}$, and were similar in all types of samples analyzed.

Table 4. Recovery and precision of the tryptophan and metabolites in the milk samples spiked at 3 concentrations $(\mathrm{n}=5)$

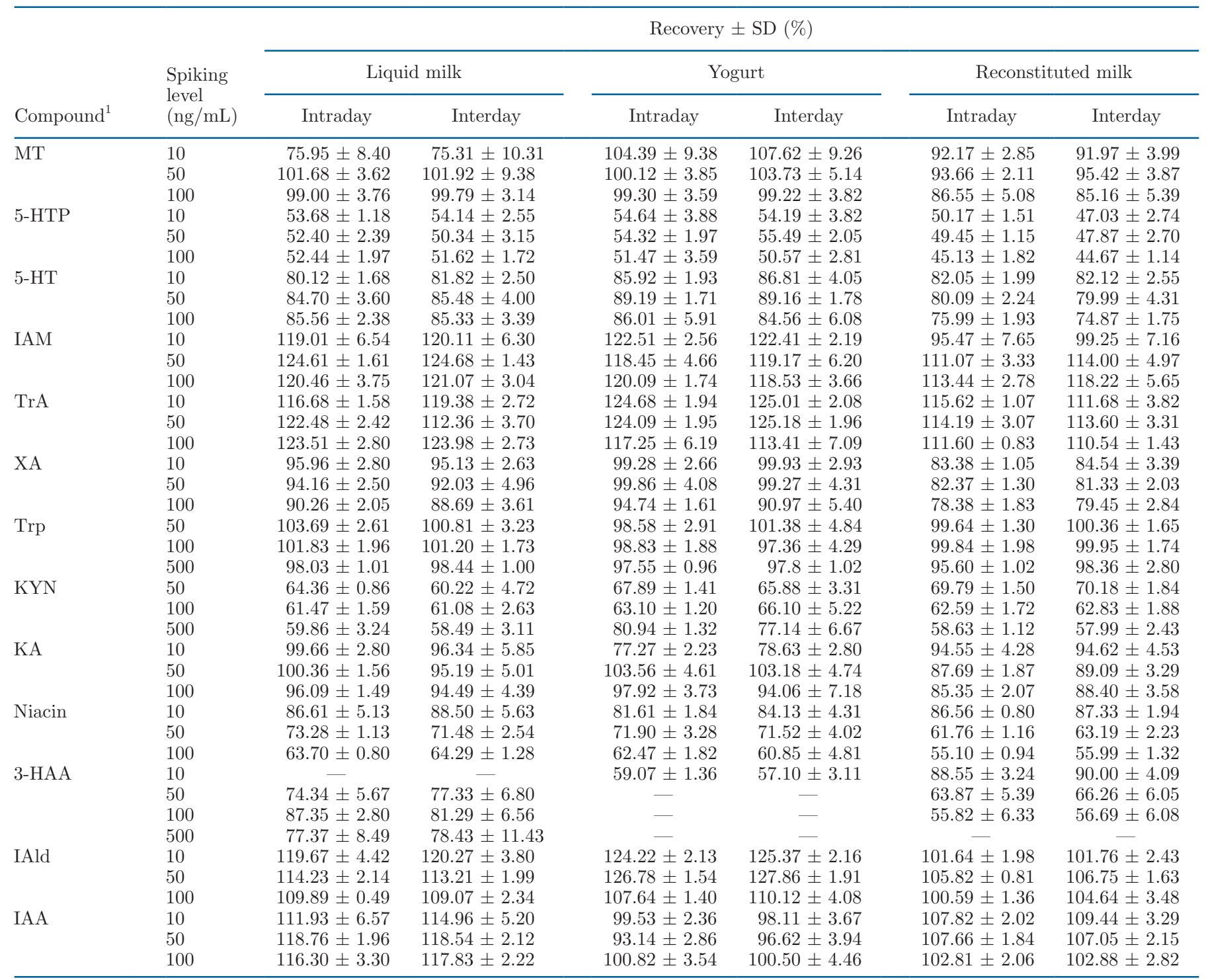

${ }^{1} \mathrm{MT}=$ melatonin; 5 -HTP $=5$-hydroxytryptophan; 5 -HT $=$ serotonin; IAM $=$ indole-3-acetamide; TrA $=$ tryptamine; 3 -HAA $=3$-hydroxyanthranilic acid; XA = xanthurenic acid; Trp = tryptophan; KYN = kynurenine; $\mathrm{KA}=$ kynurenine acid; IAId = indole-3-carboxaldehyde; IAA $=$ indole-3-acetic acid. 


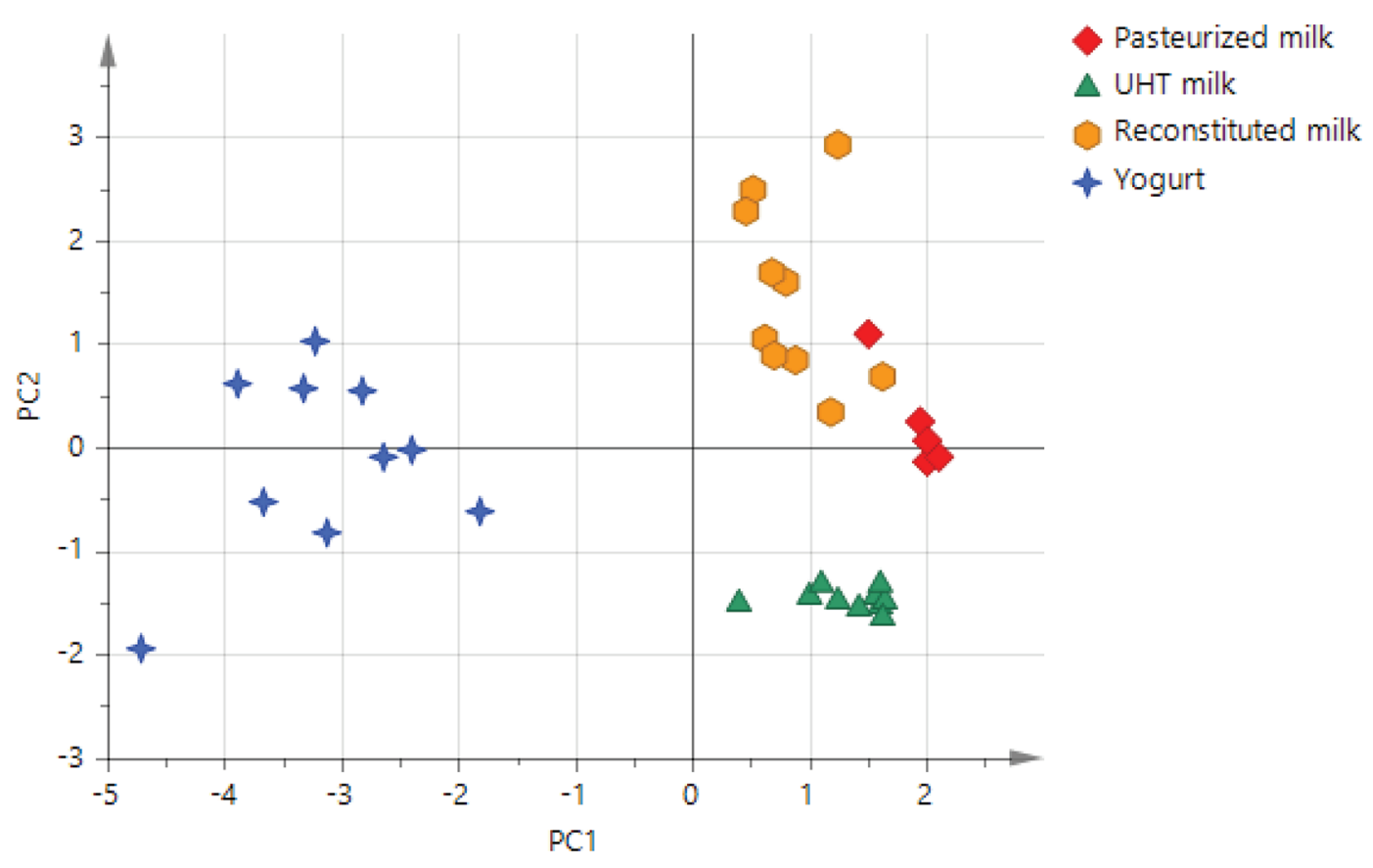

Figure 3. Partial least squares-discriminant analysis plot of tryptophan and metabolites in milk from the 4 product types (different processing technologies). Each group contains 10 samples, except for pasteurized milk, which had 5. PC $=$ principal component.

Similar levels of tryptophan were reported previously (Bertazzo et al., 2016). However, concentrations of KYN and KA differed significantly, and the lowest level of KYN (56.25 $\pm 13.69 \mathrm{ng} / 100 \mathrm{~mL}$, mean $\pm \mathrm{SD})$ was found in the UHT milk. Similarly, the content of TrA was the lowest in pasteurized and UHT milks and the highest in milk powder. The concentration of niacin ranged from 78.57 to $1421.74 \mathrm{ng} / 100 \mathrm{~mL}$ and differed significantly among milk types. 5-Hydroxytryptophan is a precursor in 5-HT biosynthesis, and we found that its concentration was much higher than that of 5-HT in milk. This trend was consistent with a previous report in the literature (Bertazzo et al., 2016). The levels of the other tryptophan metabolites were not previously

Table 5. Tryptophan and metabolite content $(\mathrm{ng} / 100 \mathrm{~mL})$ in real milk samples from the 4 processing technologies; results expressed as mean $\pm \mathrm{SD}$

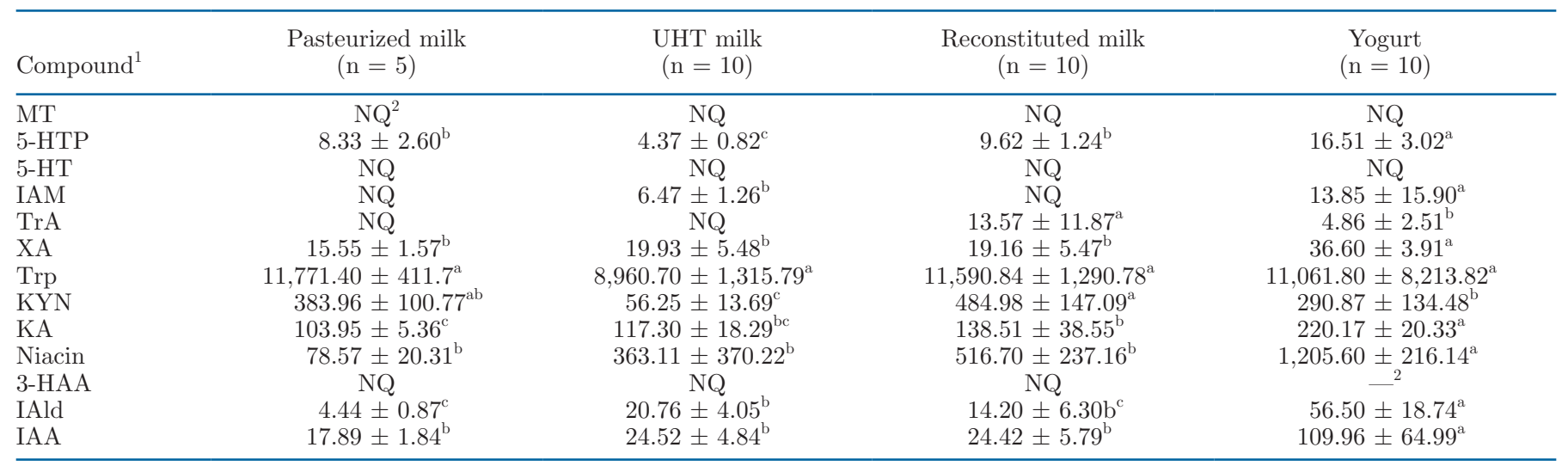

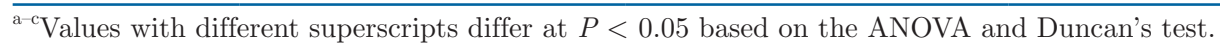

${ }^{1} \mathrm{MT}=$ melatonin; 5-HTP $=$ 5-hydroxytryptophan; 5-HT = serotonin; IAM = indole-3-acetamide; TrA = tryptamine; 3-HAA = 3-hydroxyanthranilic acid; XA = xanthurenic acid; Trp = tryptophan; KYN = kynurenine; KA = kynurenine acid; IAId = indole-3-carboxaldehyde; IAA $=$ indole-3-acetic acid.

${ }^{2}$ Below quantification limit. 
reported. 3-Hydroxyanthranilic acid is an antioxidant compound (Leipnitz et al., 2007), and its concentration ranged from 2.80 to $11.88 \mathrm{ng} / 100 \mathrm{~mL}$ in the pasteurized, UHT, and milk powder samples but it was not detected in yogurt. Indole-3-acetic acid can be synthesized by the indole pathway and further produces IAM and IAld (Tsavkelova et al., 2012). The latter can also be produced by indole-3-acetaldehyde via the neurotransmitter TrA (Agus et al., 2018). The contents of XA, IAA, IAld, and IAM were highest in yogurt and lowest in pasteurized milk.

The PLS-DA was carried out on the 4 types of milk using 9 compounds with significant differences in content. The results showed a clear distinction between yogurt and the other milk types (Figure 3 ). The presence of microorganisms such as Lactobacillus in yogurt accelerates production of tryptophan metabolites in the indole pathway, which contributes to the distinction between yogurt and other milk types. Many studies have confirmed that tryptophan is directly metabolized by microorganisms to produce IAA, TrA, and other metabolites that participate in intestinal immunity regulation (Agus et al., 2018). It is interesting to note that UHT could be clearly separated from pasteurized milk and reconstituted milk powder; this might result from the high heating temperature used in UHT milk processing. The influence of heating time is also a factor, because the pasteurized milk and reconstituted milk powder could be separated based on the PLS-DA plot (Figure 3), and heating time might explain this finding (milk powder has a longer heating time). Estévez and Luna (2017) speculated that with the increase of heating time, reactive oxygen species oxidize tryptophan into KYN and other products. We did find a higher concentration of KYN in the reconstituted milk. The effect of the shelf life is also a factor of interest and should be studied in future studies.

\section{CONCLUSIONS}

We established an effective and sensitive LC-MS/MS method to determine concentrations of tryptophan and 12 metabolites in milk. The method allowed detection of most bioactive tryptophan metabolites. Most metabolites were detected at the nanograms per milliliter level. We also discussed the effect of different milk processing methods on tryptophan metabolites. Microorganisms caused high level of IAA, IAld, and IAM in yogurt. Processing temperature caused variation of tryptophan metabolites in liquid milk. Future work should demonstrate the effect of shelf life on variations in tryptophan metabolites in milk.

\section{ACKNOWLEDGMENTS}

This work was supported by the National Key R\&D Program of China (No. 2018YFC1602300), the Natural Science Foundation of China (31871891), and Guiyang Science and Technology Bureau Special Fund for Guiyang University [GYU-KYZ (2019-2020) PT-09-05]. The authors have not stated any conflicts of interest.

\section{REFERENCES}

Agus, A., J. Planchais, and H. Sokol. 2018. Gut microbiota regulation of tryptophan metabolism in health and disease. Cell Host Microbe 23:716-724. https://doi.org/10.1016/j.chom.2018.05.003.

Arvidsson, B., N. Johannesson, A. Citterio, P. G. Righetti, and J. Bergquist. 2007. High throughput analysis of tryptophan metabolites in a complex matrix using capillary electrophoresis coupled to time-of-flight mass spectrometry. J. Chromatogr. A 1159:154-158.

Bertazzo, A., E. Ragazzi, and F. Visioli. 2016. Evolution of tryptophan and its foremost metabolites' concentrations in milk and fermented dairy products. PharmaNutrition 4:62-67. https://doi.org/10 .1016/j.phanu.2016.02.002.

Chen, Y., and G. J. Guillemin. 2009. Kynurenine pathway metabolites in humans: Disease and healthy states. Int. J. Tryptophan Res. 2:IJTR.S2097. https://doi.org/10.4137/IJTR.S2097.

Costa, F. P., S. S. Caldas, and E. G. Primel. 2014. Comparison of QuEChERS sample preparation methods for the analysis of pesticide residues in canned and fresh peach. Food Chem. 165:587-593. https://doi.org/10.1016/j.foodchem.2014.05.099.

Estévez, M., and C. Luna. 2017. Dietary protein oxidation: A silent threat to human health? Crit. Rev. Food Sci. Nutr. 57:3781-3793. https://doi.org/10.1080/10408398.2016.1165182.

Guo, H., Y. Jiao, X. Wang, T. Lu, Z. Zhang, and F. Xu. 2017. Twins labeling-liquid chromatography/mass spectrometry based metabolomics for absolute quantification of tryptophan and its key metabolites. J. Chromatogr. A 1504:83-90.

Henykova, E., H. P. Vranova, P. Amakorova, T. Pospisil, A. Zukauskaite, M. Vlckova, L. Urbanek, O. Novak, J. Mares, P. Kanovsky, and M. Strnad. 2016. Stable isotope dilution ultra-high performance liquid chromatography-tandem mass spectrometry quantitative profiling of tryptophan-related neuroactive substances in human serum and cerebrospinal fluid. J. Chromatogr. A 1437:145157.

Kato, N., T. Kojima, S. Yoshiyagawa, H. Ohta, A. Toriba, H. Nishimura, and K. Hayakawa. 2007. Rapid and sensitive determination of tryptophan, serotonin and psychoactive tryptamines by thinlayer chromatography/fluorescence detection. J. Chromatogr. A 1145:229-233.

Kocadagli, T., C. Yilmaz, and V. Gokmen. 2014. Determination of melatonin and its isomer in foods by liquid chromatography tandem mass spectrometry. Food Chem. 153:151-156.

Leipnitz, G., C. Schumacher, K. B. Dalcin, K. Scussiato, A. Solano, C. Funchal, C. S. Dutra-Filho, A. T. S. Wyse, C. M. D. Wannmacher, A. Latini, and M. Wajner. 2007. In vitro evidence for an antioxidant role of 3-hydroxykynurenine and 3-hydroxyanthranilic acid in the brain. Neurochem. Int. 50:83-94. https://doi.org/10 .1016/j.neuint.2006.04.017.

Marcos, J., N. Renau, O. Valverde, G. Aznar-Lain, I. Gracia-Rubio, M. Gonzalez-Sepulveda, L. Alberto Perez-Jurado, R. Ventura, J. Segura, and O. J. Pozo. 2016. Targeting tryptophan and tyrosine metabolism by liquid chromatography tandem mass spectrometry. J. Chromatogr. A 1434:91-101.

Mattivi, F., U. Vrhovsek, and G. Versini. 1999. Determination of indole-3-acetic acid, tryptophan and other indoles in must and wine by high-performance liquid chromatography with fluorescence detection. J. Chromatogr. A 855:227-235. https://doi.org/10.1016/ S0021-9673(99)00696-2. 
Sadok, I., K. Rachwal, and M. Staniszewska. 2019. Application of the optimized and validated LC-MS method for simultaneous quantification of tryptophan metabolites in culture medium from cancer cells. J. Pharm. Biomed. Anal. 176:112805. https://doi.org/10 $.1016 / j . j p b a .2019 .112805$.

Sonner, J. K., M. Keil, M. Falk-Paulsen, N. Mishra, A. Rehman, M. Kramer, K. Deumelandt, J. Röwe, K. Sanghvi, L. Wolf, A. von Landenberg, H. Wolff, R. Bharti, I. Oezen, T. V. Lanz, F. Wanke, Y. Tang, I. Brandao, S. R. Mohapatra, L. Epping, A. Grill, R. Röth, B. Niesler, S. G. Meuth, C. A. Opitz, J. G. Okun, C. Reinhardt, F. C. Kurschus, W. Wick, H. B. Bode, P. Rosenstiel, and M. Platten. 2019. Dietary tryptophan links encephalogenicity of autoreactive $\mathrm{T}$ cells with gut microbial ecology. Nat. Commun. 10:4877. https://doi.org/10.1038/s41467-019-12776-4.

Soto, M. E., A. M. Ares, J. Bernal, M. J. Nozal, and J. L. Bernal. 2011. Simultaneous determination of tryptophan, kynurenine, kynurenic and xanthurenic acids in honey by liquid chromatography with diode array, fluorescence and tandem mass spectrometry detection. J. Chromatogr. A 1218:7592-7600. https://doi.org/10.1016/ j.chroma.2011.06.105.

Tsavkelova, E., B. Oeser, L. Oren-Young, M. Israeli, Y. Sasson, B. Tudzynski, and A. Sharon. 2012. Identification and functional characterization of indole-3-acetamide-mediated IAA biosynthesis in plant-associated Fusarium species. Fungal Genet. Biol. 49:48 57. https://doi.org/10.1016/j.fgb.2011.10.005.
Turski, M. P., M. Turska, T. Kocki, W. A. Turski, and P. Paluszkiewicz. 2015. Kynurenic acid content in selected culinary herbs and spices. J. Chem. 2015:617571. https://doi.org/10.1155/2015/ 617571.

Turski, M. P., M. Turska, W. Zgrajka, M. Bartnik, T. Kocki, and W. A. Turski. 2011. Distribution, synthesis, and absorption of kynurenic acid in plants. Planta Med. 77:858-864. https://doi.org/10 $.1055 / \mathrm{s}-0030-1250604$.

Wilkowska, A., and M. Biziuk. 2011. Determination of pesticide residues in food matrices using the QuEChERS methodology. Food Chem. 125:803-812. https://doi.org/10.1016/j.foodchem.2010.09 .094 .

Yllmaz, C., and V. Gokmen. 2018. Determination of tryptophan derivatives in kynurenine pathway in fermented foods using liquid chromatography tandem mass spectrometry. Food Chem. 243:420-427. https://doi.org/10.1016/j.foodchem.2017.10.004.

\section{ORCIDS}

Meicheng Su ㄴ https://orcid.org/0000-0003-1865-0071

Gang Chen $\odot$ https://orcid.org/0000-0002-2451-8299 